\title{
Modelling of single degree of freedom SMA oscillators by using rheological schemes
}

\author{
Artur Zbiciak ${ }^{1}$, and Kacper Wasilewski ${ }^{{ }^{*}}$ \\ ${ }^{1}$ Warsaw University of Technology, Faculty of Civil Engineering, 16 Lecha Kaczyńskiego Str., 00- \\ 637 Warsaw, Poland
}

\begin{abstract}
The article describes the approach to modelling of single degree of freedom SMA oscillators by using rheological schemes. Certain sets of rheological components are presented and their influence on the oscillator response is examined. Regarding the field of civil engineering, the devices incorporating SMA elements mostly find applications in mitigation of natural disaster hazards, such as earthquakes. The promising results of applications are possible due to unique properties of SMA, such as shape memory effect (recovering of relatively high strains while material is heated) and superelasticity (recovering of strains upon load removal). The most common approach to the formulation of SMAs constitutive relations is a thermomechanical modelling, in which constitutive equations are dependent on internal state variables. One of the advantages of the phenomenological modelling approach presented in the article is a possibility of formulation of constitutive relationships as a set of explicit differential equations. Such system of equations can be easily implemented in mathematical software or in the commercial FEM codes as a user's subroutines. As an example of numerical application of presented approach, the simple one-dimensional oscillator is used in order to solve the case of forced vibrations of a cantilever with embedded SMA reinforcement.
\end{abstract}

\section{Introduction}

Main fields of the shape memory alloys (SMAs) application in civil engineering are oriented on mitigation of natural disaster hazards, such as significant ground motions related to earthquakes. It can be achieved due to unique characteristics of SMA such as shape memory effects and superelasticity. Shape memory effect is a possibility of recovering from the deformed shape while material is heated. Superelasticity is an ability to return from a relatively high strains to original shape upon a load removal [1].

The characteristics of SMA are associated with the phase transformation. In case of the shape memory effect phase transformation is a result of heating and in case of superelasticity it is induced by external stresses in isothermal conditions, above certain temperature $\left(A_{f}\right)$. The hysteretic loop obtained during the uniaxial tensions test [2] is

\footnotetext{
* Corresponding author: k.wasilewski@il.pw.edu.pl
} 
presented in Fig. 1. At the beginning of the test sample is in austenite phase $(A)$ and the load application induces a transformation to the de-twined martensite $(M)$. This phase transformation is illustrated as an arrow no. 1 in Fig. 1. Upon unloading, a reverse phase transformation (arrow no. 2 in Fig. 1) occurs and the material is back in original austenite phase. The revers transformation results with full strain recovery [3].

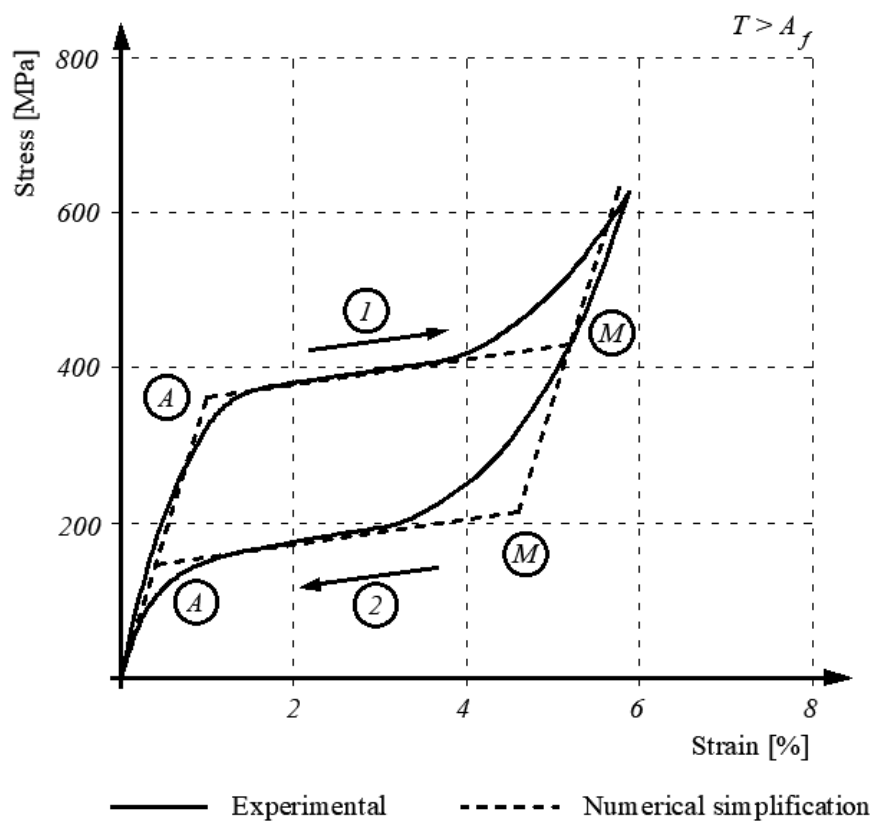

Fig. 1. Results of uniaxial tension test of SMA [2] and its numerical simplification.

From the point of view of application of SMA in a field of structural engineering, superelasticity phenomenon is particularly interesting. It is one of the main issues of the material models, used for numerical analysis of structures with SMA applied.

This article proposes a representation of SMA oscillator by rheological schemes. Authors analyse certain sets of rheological components and their influence on the oscillator response. Later, the simple one-dimensional oscillator is used in order to solve the example of forced vibrations of a cantilever with embedded SMA reinforcement.

\section{Oscillators with SMA}

Topic of one-dimensional oscillators with SMA where studied earlier, for example by Savi and Braga [4], Feng and Li [5], Machado [6] or Sitnikova et al.[7]. All of those authors implemented well known thermomechanical approach to formulation of SMA's constitutive relations. In such an approach, constitutive equations are dependent on internal state variables for example - the martensitic volume fraction $(\xi)$ and the transformation strain $\left(\varepsilon^{t}\right)$.

The SMA's material model presented by authors of this paper is based on the analysis of the rheological schemes composed of different components. It is a continuation of work of Grzesikiewicz and Zbiciak [8-12] and Wasilewski and Zbiciak [13]. 


\subsection{Basic rheological components}

The schematic representation of the SMA oscillator is shown in Fig. 2a, where the phenomenological model of SMA is given as a, so called, black box. The rheological models, that interpret the black box are composed of four basic components:

- linear elastic spring (Hook's material) - Fig. 2b,

- rigid, perfectly plastic body (Saint-Venant's body) - Fig. 2c,

- rigid, perfectly elastic body (Hencky's body) - Fig. 2d,

- elastic body with limited strains (locking material) - Fig. 2e.

a)

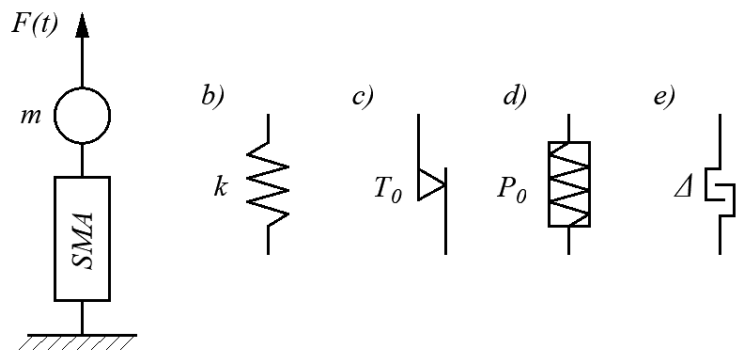

Fig. 2. Schematic representation of SMA oscillator and basic components used in rheological models with symbols for their mechanical characteristics.

Proper configuration of perfectly plastic and perfectly elastic body creates a rheological model that will represent the phenomenon of superelasticity of SMA. The perfectly plastic body is responsible for energy dissipation of the structure, while the perfectly elastic, along with linear elastic springs, are responsible for energy accumulation.

\subsection{Rheological models}

The different sets of rheological components were analysed as an interpretation of SMA's black box in Fig. 2a. The simplest of the considered rheological schemes was composed of elastic spring and configuration of perfectly elastic with perfectly plastic body (Fig. 3). The hysteretic loop of this system clearly shows superelasticity phenomenon. At the beginning of the loading the displacement of the mass is linear and depends on the spring stiffness $\left(k_{2}\right)$. When the force reaches a certain level, equals to $P_{0}+T_{0}$, a characteristic plateau, related to phase transformation, appears. This model does not present hardening during the phase transformation. The unloading path is parallel to the loading one and contains another plateau, related to revers phase transformation, at the level of force to be equal $P_{0}-T_{0}$, and reaches the value of 0 upon further force decrease. 

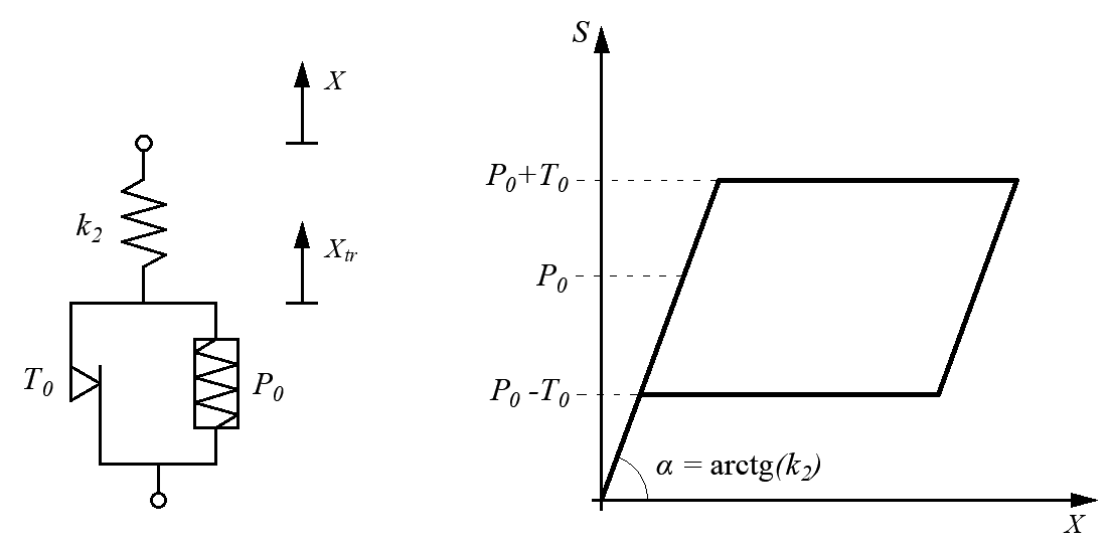

Fig. 3. Basic rheological scheme of SMA material and the graph of hysteretic loop.

The equation of motion of the oscillator is given by the following differential equation

$$
\ddot{X}(t)=\frac{1}{m}[F(t)-S(t)]
$$

where $F(t)$ is external excitation, $S(t)$ is a force response of rheological model and $X$ is a $m$ mass displacement. This equation is of an universe nature and can be applied to all of the considered oscillators.

The internal force in SMA black box is given by

$$
S(t)=k_{2}\left(X(t)-X_{t r}(t)\right)
$$

The rate of deformation of the phase transformation is given by:

$$
\dot{X}_{t r}=f_{S M A}\left(\dot{X}, X, X_{t r}\right) \text {, }
$$

where

$$
f_{S M A}= \begin{cases}\dot{X} & \text { if }|S|=P_{0}+T_{0} \text { and } S \dot{X}>0 \\ \dot{X} & \text { if }|S|=P_{0}-T_{0} \text { and } S \dot{X}<0 \text { and } S X_{t r}>0 \\ 0 & \text { otherwise }\end{cases}
$$

The aim of the second rheological scheme was to represent the hardening nature of the phase transformation from austenite to de-twined martensite (the arrow no. 1 in Fig. 1). It was obtained by the addition of the second parallel spring (Fig. 4). The change of rheological scheme keeps the validity of equation (1), but results in the modification of the force response equation (2), in the following manner

$$
\begin{aligned}
& S(t)=S_{1}(t)+S_{2}(t) \\
& S_{1}(t)=k_{1} X(t) \\
& S_{2}(t)=k_{2}\left(X(t)-X_{t r}(t)\right)
\end{aligned}
$$

Moreover, the internal force $S$ in equation (3) should be replaced by $S_{2}$. 

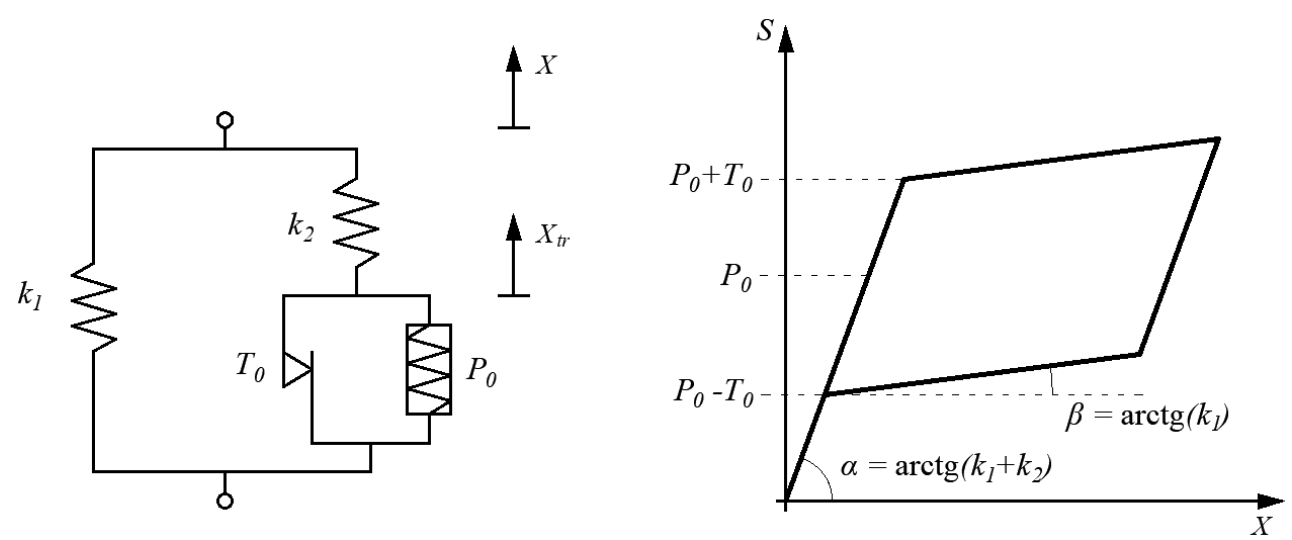

Fig. 4. Rheological scheme of SMA material with hardening and the graph of hysteretic loop.

The third rheological scheme represents, in the addition to the second one, the phenomenon of the locking that occurs after the phase transformation while the loads are still increasing. This result was obtained by addition of an elastic body with limited strains (Fig. 5). For this rheological schemes equations (1) and (4) are still valid, but the equation (3) transforms to

$$
\dot{X_{t r}}=f_{S M A}\left(\dot{X}, X, X_{t r}\right),
$$

where

$$
f_{S M A}= \begin{cases}\dot{X} & \text { if }\left|S_{2}\right|=P_{0}+T_{0} \text { and } S_{2} \dot{X}>0 \text { and }\left|X_{t r}\right|<\Delta \\ \dot{X} & \text { if }\left|S_{2}\right|=P_{0}-T_{0} \text { and } S_{2} \dot{X}<0 \text { and } S_{2} X_{t r}>0 \\ 0 & \text { otherwise }\end{cases}
$$
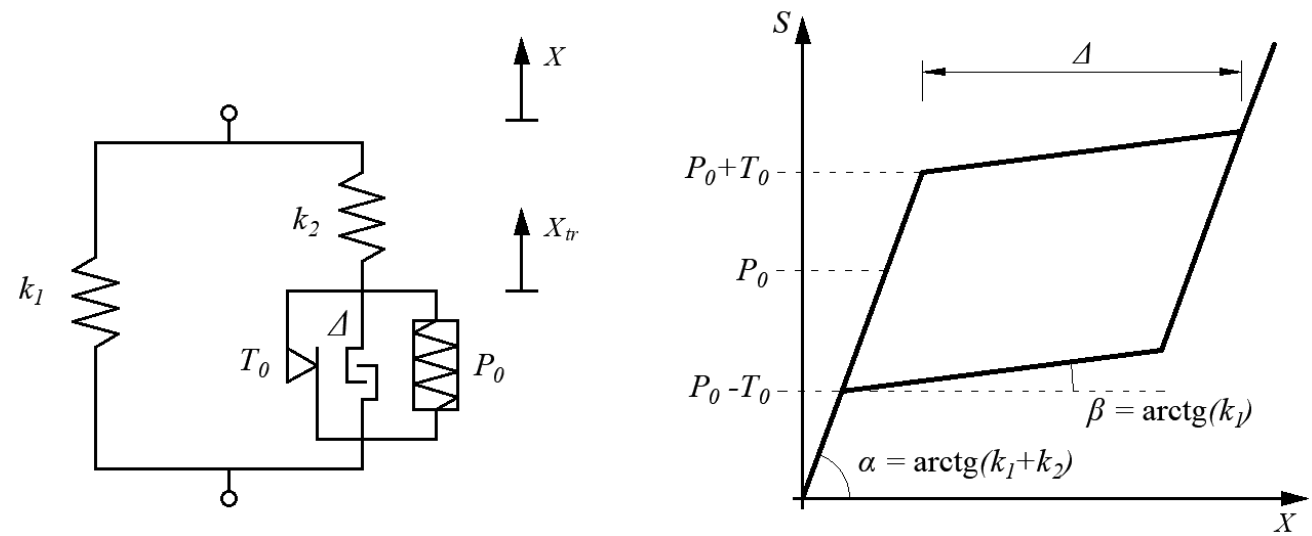

Fig. 5. Rheological scheme of SMA material with hardening and locking and the graph of hysteretic loop.

\section{Numerical application}

Let us consider a cantilever of the length $l$ with a mass $m$ located at the end and the vertical load $P(t)$ applied to the mass (Fig. 6). The cantilever is composed of $n$ SMA rods 
embedded in soft matrix (the influence of the matrix to the stiffness of the cantilever is neglected).
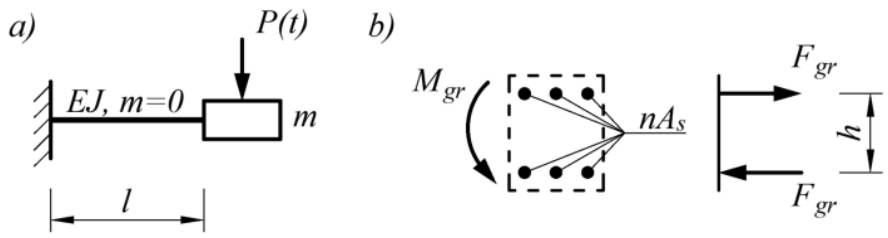

Fig. 6. Cantilever subjected to vertical force excitation.

The geometrical variables are presented in the Table 1 along with mechanical parameters estimated based on experimental results presented by Auricchio and Sacco [14].

Table 1 Geometrical and mechanical parameters.

\begin{tabular}{|c|c|c|c|c|c|c|c|c|c|}
\hline \multicolumn{9}{|c|}{ Geometrical variables } & \multicolumn{5}{c|}{ Mechanical parameters } \\
\hline$m$ & $n$ & $h$ & $l$ & $A_{s}$ & $E_{1}$ & $E_{2}$ & $\sigma_{p l}$ & $\sigma_{p e}$ & $\Delta$ \\
\hline$[\mathrm{kg}]$ & {$[-]$} & {$[\mathrm{mm}]$} & {$[\mathrm{mm}]$} & {$\left[\mathrm{mm}^{2}\right]$} & {$[\mathrm{GPa}]$} & {$[\mathrm{GPa}]$} & {$[\mathrm{MPa}]$} & {$[\mathrm{MPa}]$} & {$[\mathrm{mm}]$} \\
\hline 100 & 6 & 150 & 1000 & 314 & 50 & 5 & 140 & 380 & 80 \\
\hline
\end{tabular}

Based on the values shown in Table 1 the required characteristics of the components of rheological model visualised in Fig. 5, where calculated and given in Table 2.

Table 2 Parameters of rheological model of SMA.

\begin{tabular}{|c|c|c|c|c|}
\hline$k_{1}$ & $k_{2}$ & $T_{0}$ & $P_{0}$ & $\Delta$ \\
\hline$[\mathrm{N} / \mathrm{mm}]$ & {$[\mathrm{N} / \mathrm{mm}]$} & {$[\mathrm{N}]$} & {$[\mathrm{N}]$} & {$[\mathrm{mm}]$} \\
\hline 159,75 & 1437,75 & 19792 & 53721,2 & 80 \\
\hline
\end{tabular}

The applied harmonic load is given by

$$
F(t)=A \cdot \sin (\omega t)
$$

where $A=50 \mathrm{kN}$ and $\omega=4 \frac{\mathrm{rad}}{\mathrm{s}} \cong 0,6366 \mathrm{~Hz}$.

The presented equations (1), (4) and (5) were implemented in Wolfram Mathematica software. The set of differential equations was numerically solved applying NDSolve function by incorporation of forward Euler (ExplicitEuler) method. The results are presented below as a set of graphs showing the time histories of the mass displacement $(X)$ and the phase transformation displacement $\left(X_{t r}\right)$ (Fig. 7) as well as rate of displacement ( $\dot{X}$ ) (Fig. 8). The hysteretic loop of the analysed oscillator is depicted in Fig. 9. 


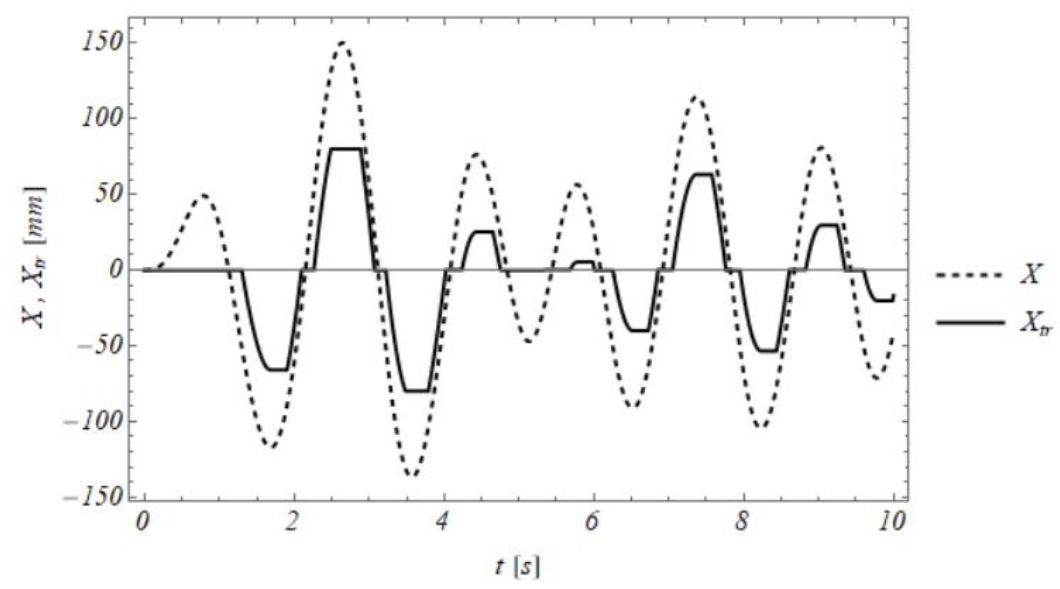

Fig. 7. Time histories of the mass displacement $(X)$ and the phase transformation displacement $\left(X_{t r}\right)$.

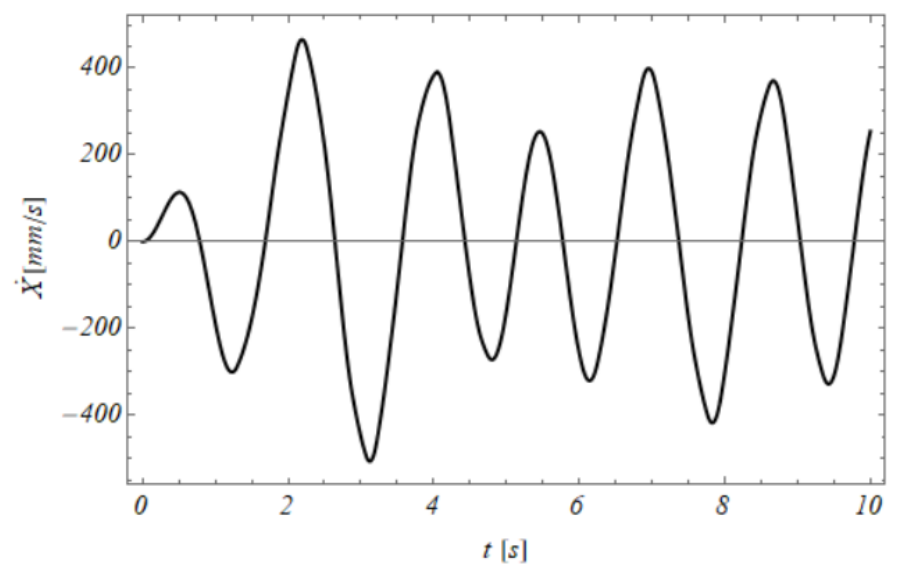

Fig. 8. Time history of rate of displacement $(\dot{X})$.

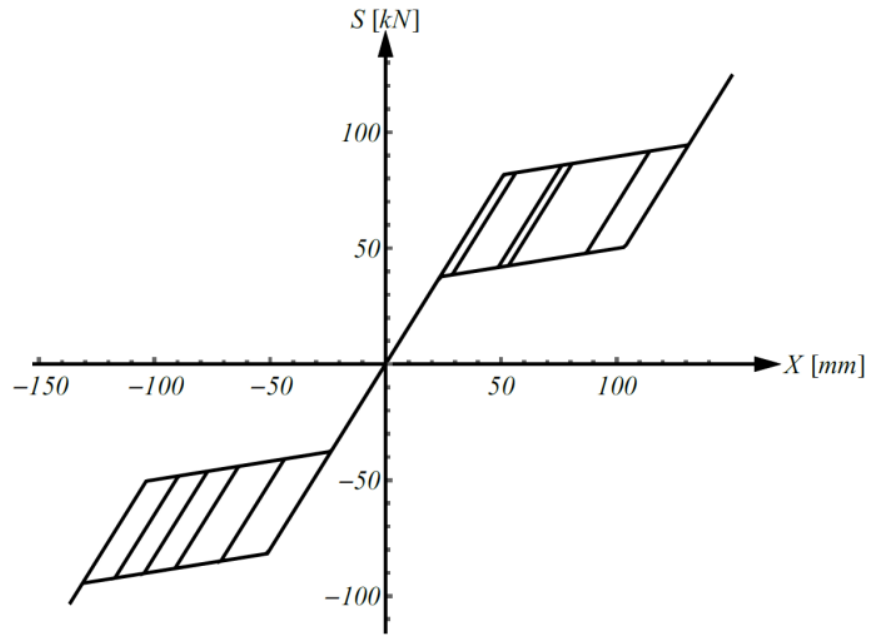

Fig. 9. Hysteretic loop of the analysed SMA oscillator. 


\section{Conclusions}

Results of the presented numerical example show the validity of the formulation. It should be emphasised that the proposed methodology of the formulation of SMA constitutive equations is based on original rheological schemes. It results in explicit form of differential equations defining all of the presented models. Such system of equations can be easily implemented in mathematical software.

The presented formulation of constitutive relations could be exploit in the dynamic analysis of a vibration of different structural elements (such as beams, frames or trusses). The equations could be directly implemented in the commercial FEM codes as a user's subroutines (e.g. UMAT and VUMAT subroutines in Abaqus [8-11]).

\section{References}

1. J. Mohd Jani, M. Leary, A. Subic, M.A. Gibson, Mater. Des., 56,1078-1113, (2014)

2. G. Faiella, V. Antonucci, in Shape Mem. Alloy Eng., 57-77, (Elsevier Ltd, 2015)

3. V. Antonucci, A. Martone, in Shape Mem. Alloy Eng., 33-56, (Elsevier Ltd, 2015)

4. M.A. Savi, A.M.B. Braga, J. Brazilian Soc. Mech. Sci. Eng., 15,1-20, (1993)

5. Z.C. Feng, D.Z. Li, (1996)

6. L. Machado, (Texas A\&M University, 2007)

7. E. Sitnikova, E. Pavlovskaia, J. Ing, M. Wiercigroch, Smart Mater. Struct., 21, (2012)

8. W. Grzesikiewicz, A. Zbiciak, Comput. Syst. Aided Sci. Eng. Work Transp. Mech. Electr. Eng., 122,159-164, (2008)

9. W. Grzesikiewicz, A. Wakulicz, A. Zbiciak, Model. Inżynierskie, 105-110, (2009)

10. W. Grzesikiewicz, A. Wakulicz, A. Zbiciak, Int. J. Non. Linear. Mech., 46,870-876, (2011)

11. W. Grzesikiewicz, A. Zbiciak, in Proc. 15th French-Polish Semin. Mech., 138-143, (2007)

12. A. Zbiciak, Int. J. Mech. Sci., 52,56-64, (2010)

13. K. Wasilewski, A. Zbiciak, in 3rd Int. Conf. Prot. Hist. Constr., (Mazzolani, F. M.) 129-130, (2017)

14. F. Auricchio, E. Sacco, Int. J. Non. Linear. Mech., 32,1101-1114, (1997) 